TRANSACTIONS OF THE

AMERICAN MATHEMATICAL SOCIETY

Volume 353 , Number 7 , Pages $2843-2857$

S 0002-9947(01)02796-9

Article electronically published on March 12, 2001

\title{
SPECTRAL LIFTING IN BANACH ALGEBRAS AND INTERPOLATION IN SEVERAL VARIABLES
}

\author{
GELU POPESCU
}

\begin{abstract}
Let $\mathcal{A}$ be a unital Banach algebra and let $J$ be a closed two-sided ideal of $\mathcal{A}$. We prove that if any invertible element of $\mathcal{A} / J$ has an invertible lifting in $\mathcal{A}$, then the quotient homomorphism $\Phi: \mathcal{A} \rightarrow \mathcal{A} / J$ is a spectral interpolant. This result is used to obtain a noncommutative multivariable analogue of the spectral commutant lifting theorem of Bercovici, Foiaş, and Tannenbaum. This yields spectral versions of Sarason, Nevanlinna-Pick, and Carathéodory type interpolation for $F_{n}^{\infty} \bar{\otimes} B(\mathcal{K})$, the WOT-closed algebra generated by the spatial tensor product of the noncommutative analytic Toeplitz algebra $F_{n}^{\infty}$ and $B(\mathcal{K})$, the algebra of bounded operators on a finite dimensional Hilbert space $\mathcal{K}$. A spectral tangential commutant lifting theorem in several variables is considered and used to obtain a spectral tangential version of the Nevanlinna-Pick interpolation for $F_{n}^{\infty} \bar{\otimes} B(\mathcal{K})$.

In particular, we obtain interpolation theorems for matrix-valued bounded analytic functions on the open unit ball of $\mathbb{C}^{n}$, in which one bounds the spectral radius of the interpolant and not the norm.
\end{abstract}

\section{INTRODUCTION AND PRELIMINARIES}

Let $\mathbb{D}$ denote the unit disc in the complex plane, let $z_{1}, \ldots, z_{k} \in \mathbb{D}$ be given distinct points, and $F_{1}, \ldots, F_{k}$ be complex $m \times m$ matrices. The classical NevanlinnaPick problem $[\mathrm{N},[\mathrm{P}]$ consists in finding necessary and sufficient conditions for the existence of an analytic $m \times m$ matrix-valued function $F(z)$ with $F\left(z_{j}\right)=F_{j}$ $(1 \leq j \leq k)$ and such that $\|F\|_{\infty} \leq 1$.

Motivated by problems in control engineering, such as the design of feedback control systems in the presence of parameter uncertainty, Bercovici, Foiaş, and Tannenbaum proved in [BFT] a spectral generalization of the commutant lifting theorem [SzF1], and obtained a spectral version of the Nevanlinna-Pick problem, in which the infinity norm is replaced by

$$
\rho(F):=\sup \left\{\|F(z)\|_{\mathrm{sp}}: z \in \mathbb{D}\right\}
$$

$\left(\|A\|_{\mathrm{sp}}\right.$ denotes the spectral radius of an operator $\left.A\right)$.

The tangential Nevanlinna-Pick problem considered by Fedcina $[\mathrm{F}]$ is to find $F \in H^{\infty}(\mathbb{D}) \otimes \mathbb{C}^{m}$ with $F\left(z_{j}\right) u_{j}=v_{j}, j=1, \ldots, k$, and $\|F\|_{\infty} \leq 1$, where $z_{j} \in \mathbb{D}$ and $u_{j}, v_{j} \in \mathbb{C}^{m}$ are prescribed. The spectral tangential Nevanlinna-Pick interpolation problem, considered by Bercovici and Foias $[\overline{B F}$, is to find such an $F$ for which $\rho(F)<1$. This type of interpolation was also motivated by certain control engineering applications.

Received by the editors December 22, 1998 and, in revised form, October 4, 1999.

2000 Mathematics Subject Classification. Primary 47L25, 47A57, 47A20; Secondary 30E05.

Partially supported by NSF Grant DMS-9531954. 
In this paper we find noncommutative multivariable analogues of the abovementioned results obtained by Bercovici, Foiaş, and Tannenbaum (see [BFT] and [BF]) for the noncommutative analytic Toeplitz algebra $F_{n}^{\infty}$. In particular, we obtain interpolation results (see Corollary 3.7 and Corollary 4.3) for matrix-valued bounded analytic functions on the open unit ball of $\mathbb{C}^{n}$, in which one bounds the spectral radius of the interpolant and not the norm.

We expect these results to play a role in multivariable control and systems theory, as it does in the case $n=1$. We mention the papers [BV] and [B] for recent results in multivariable linear systems.

We need to recall some facts concerning the noncommutative analytic Toeplitz algebra $F_{n}^{\infty}$ and its connection with the function theory on the open unit ball of $\mathbb{C}^{n}$. Let $F^{2}\left(H_{n}\right)=\mathbb{C} 1 \oplus \bigoplus_{m \geq 1} H_{n}^{\otimes m}$ be the full Fock space on $n$ generators, where $H_{n}$ is an $n$-dimensional complex Hilbert space with orthonormal basis $\left\{e_{1}, e_{2}, \ldots, e_{n}\right\}$ if $n$ is finite, and $\left\{e_{1}, e_{2}, \ldots\right\}$ if $n=\infty$. For each $i=1,2, \ldots$, define the left creation operator by $S_{i} \xi:=e_{i} \otimes \xi, \xi \in F^{2}\left(H_{n}\right)$.

We shall denote by $\mathcal{P}$ the set of all $p \in F^{2}\left(H_{n}\right)$ which are finite sums of tensor monomials. Define $F_{n}^{\infty}$ as the set of all $g \in F^{2}\left(H_{n}\right)$ such that

$$
\|g\|_{\infty}:=\sup \left\{\|g \otimes p\|_{F^{2}\left(H_{n}\right)}: p \in \mathcal{P},\|p\|_{F^{2}\left(H_{n}\right)} \leq 1\right\}<\infty .
$$

We denote by $\mathcal{A}_{n}$ the closure of $\mathcal{P}$ in $\left(F_{n}^{\infty},\|\cdot\|_{\infty}\right)$. The Banach algebra $F_{n}^{\infty}$ (resp. $\mathcal{A}_{n}$ ) can be viewed as a noncommutative analogue of the Hardy space $H^{\infty}(\mathbb{D})$ (resp. disc algebra $A(\mathbb{D})$ ); when $n=1$ they coincide.

In [Po7, Theorem 3.1] we proved that $\mathcal{A}_{n}$ is completely isometrically isomorphic to the norm-closed algebra generated by any sequence $V_{1}, \ldots, V_{n}$ of isometries with $V_{1} V_{1}^{*}+\cdots+V_{n} V_{n}^{*} \leq I$, and the identity. It follows from [P05, Theorem 4.3] that the noncommutative analytic Toeplitz algebra $F_{n}^{\infty}$ can be identified with the WOTclosed algebra generated by the left creation operators $S_{1}, \ldots S_{n}$, and the identity. The algebras $F_{n}^{\infty}$ and $\mathcal{A}_{n}$ were introduced by the author in Po3] in connection with a noncommutative von Neumann inequality, and have been studied in several papers [Po2], [Po5], [Po6], [Po7], [Po9], [ArPo1], and recently in [DP1], [DP2], ArPo2], [DP3], and [Po8].

We established a strong connection between the algebra $F_{n}^{\infty}$ and the function theory on the open unit ball $\mathbb{B}_{n}$ of $\mathbb{C}^{n}$ through the noncommutative von Neumann inequality [Po3] (see also Po5], Po7], and [Po9]). In particular, we proved that there is a completely contractive homomorphism

$$
\Phi: F_{n}^{\infty} \rightarrow H^{\infty}\left(\mathbb{B}_{n}\right), \quad f\left(S_{1}, \ldots, S_{n}\right) \mapsto f\left(\lambda_{1}, \ldots, \lambda_{n}\right),
$$

where $\left(\lambda_{1}, \ldots, \lambda_{n}\right) \in \mathbb{B}_{n}$. A characterization of the analytic functions in the range of the map $\Phi$ was obtained in [ArPo2] and [DP3]. W. Arveson proved that $\Phi$ is not surjective [Arv and the functions in its range are the multipliers of a certain function Hilbert space. In [ArPo2], DP3], it was proved that $F_{n}^{\infty} / \operatorname{ker} \Phi$ is an operator algebra which can be identified with $\mathcal{W}_{n}^{\infty}:=\left.P_{F_{s}^{2}} F_{n}^{\infty}\right|_{F_{s}^{2}}$, the compression to the symmetric Fock space $F_{s}^{2} \subseteq F^{2}\left(H_{n}\right)$. In Po8], Po9], Arv, ArPo2, DP3], AMc], and [BTV], a good case is made that the appropriate commutative multivariable analogue of $H^{\infty}(\mathbb{D})$ is the algebra $\mathcal{W}_{n}^{\infty}$, which is the WOT-closed algebra generated by $B_{i}:=\left.P_{F_{s}^{2}} S_{i}\right|_{F_{s}^{2}}, i=1, \ldots, n$, and the identity. In this paper, we provide further evidence that $F_{n}^{\infty}$ (resp. $\mathcal{W}_{n}^{\infty}$ ) is a noncommutative (resp. commutative) multivariate analogue of $H^{\infty}(\mathbb{D})$. 
Let $\mathcal{A}$ be a unital Banach algebra and denote by $\operatorname{Inv}(\mathcal{A})$ the group of invertible elements of $\mathcal{A}$. Given $a \in \mathcal{A}$, we define the $\mathcal{A}$-spectral radius of $a$ by setting

$$
\rho_{\mathcal{A}}(a):=\inf \left\{\left\|x a x^{-1}\right\|: x \in \operatorname{Inv}(\mathcal{A})\right\} .
$$

Since the spectral radius of $a \in \mathcal{A}$ is $\|a\|_{\mathrm{sp}}=\lim _{n \rightarrow \infty}\left\|a^{n}\right\|^{1 / n}$, it is clear that $\|a\|_{\mathrm{sp}}=\left\|x a x^{-1}\right\|_{\mathrm{sp}}$ for any $x \in \operatorname{Inv}(\mathcal{A})$. Now, it is easy to see that

$$
\|a\|_{\mathrm{sp}} \leq \rho_{\mathcal{A}}(a) \leq\|a\|
$$

for any $a \in \mathcal{A}$. Note that if $\mathcal{A}=B(\mathcal{H})$ (or $\mathcal{A}$ is any $C^{*}$-subalgebra of $B(\mathcal{H})$ ) then $\|a\|_{\mathrm{sp}}=\rho_{\mathcal{A}}(a)$ (see $[\mathbb{\mathrm { R }})$. There are some other examples of Banach algebras such that $\|a\|_{\mathrm{sp}}=\rho_{\mathcal{A}}(a)$ for any $a \in \mathcal{A}$. It was proved in $[\mathrm{BFT}]$ that this equality holds if $\mathcal{A}$ is the commutant of an isometry (resp. normal operator) on a Hilbert space.

Let $\mathcal{A}, \mathcal{B}$ be unital Banach algebras, and $\Phi: \mathcal{A} \rightarrow \mathcal{B}$ be a unital contractive homomorphism. We say that $\Phi$ is a quotient interpolant if

$$
\|b\|=\inf \{\|a\|: a \in \mathcal{A}, \Phi(a)=b\}
$$

for any $b \in \mathcal{B}$. We say that $b \in \mathcal{B}$ with $\rho_{\mathcal{B}}(b)<1$ has a spectral lifting if there exists $a \in \mathcal{A}$ such that $\Phi(a)=b$ and $\rho_{\mathcal{A}}(a)<1$. The homomorphism $\Phi$ is called a spectral interpolant if any $b \in \mathcal{B}$ has a spectral lifting.

Problem. Let $\Phi: \mathcal{A} \rightarrow \mathcal{B}$ be a unital contractive homomorphism which is also a quotient interpolant. When is $\Phi$ a spectral interpolant?

We show, in Section 2, that this problem has a positive answer if $\operatorname{Inv}(\mathcal{B}) \subseteq$ $\Phi(\operatorname{Inv}(\mathcal{A}))$. This relation holds, for example, if the group of invertible elements of $\mathcal{B}$ is connected (in particular, if $\mathcal{B}$ is finite dimensional or equal to $B(\mathcal{H})$ ).

The results of Section 2 are used in Section 3 to obtain a noncommutative multivariable analogue (see Theorem 3.1) of the spectral commutant lifting theorem of Bercovici-Foiaş-Tannenbaum. This yields spectral versions of Sarason ([S] $)$, Nevanlinna-Pick, and Carathéodory type interpolation for $F_{n}^{\infty} \bar{\otimes} B(\mathcal{K})$, the WOTclosed algebra generated by the spatial tensor product of the noncommutative analytic Toeplitz algebra $F_{n}^{\infty}$ and $B(\mathcal{K})$, the algebra of bounded operators on a finite dimensional Hilbert space $\mathcal{K}$.

In Section 4, we obtain a spectral tangential commutant lifting theorem in several variables (see Theorem 4.1). This leads to a spectral tangential Nevanlinna-Pick interpolation for $F_{n}^{\infty} \bar{\otimes} B(\mathcal{K})$ (see Theorem 4.2).

Problems concerning the optimal solutions to these spectral interpolation problems in several variables, and explicit algorithm for finding the optimal interpolants will be considered in a future paper.

We would like to thank the referee for helpful comments on the results of this paper.

\section{Spectral lifting in Banach algebras}

The notation and definitions from Section 1 are used throughout the paper. Let $\mathcal{A}, \mathcal{B}$ be unital Banach algebras and let $\Phi: \mathcal{A} \rightarrow \mathcal{B}$ be a unital contractive homomorphism. We call $\Phi$ a norm preserving interpolant if for any $b \in \mathcal{B}$ there exists $a \in \mathcal{A}$ such that $\Phi(a)=b$ and $\|a\|=\|b\|$. Notice that any norm preserving interpolant is a quotient interpolant. Examples of norm preserving interpolants will be presented in Section 3. 
Theorem 2.1. Let $\mathcal{A}, \mathcal{B}$ be unital Banach algebras and let $\Phi: \mathcal{A} \rightarrow \mathcal{B}$ be a unital contractive homomorphism with the property that $\operatorname{Inv}(\mathcal{B}) \subseteq \Phi(\operatorname{Inv}(\mathcal{A}))$ and

$$
\|b\|=\inf \{\|a\|: a \in \mathcal{A}, \Phi(a)=b\}
$$

for any $b \in \mathcal{B}$. Then

$$
\rho_{\mathcal{B}}(b)=\inf \left\{\rho_{\mathcal{A}}(a): a \in \mathcal{A}, \Phi(a)=b\right\}
$$

for any $b \in \mathcal{B}$. In particular, $\Phi$ is a spectral interpolant.

Proof. Let $b \in \mathcal{B}$ and $a \in \mathcal{A}$ with $\Phi(a)=b$. Since $\Phi$ is a contractive homomorphism and $\Phi(\operatorname{Inv}(\mathcal{A})) \subseteq \operatorname{Inv}(\mathcal{B})$ we have

$$
\begin{aligned}
\rho_{\mathcal{A}}(a) & =\inf \left\{\left\|w a w^{-1}\right\|: w \in \operatorname{Inv}(\mathcal{A})\right\} \\
& \geq \inf \left\{\left\|\Phi\left(w a w^{-1}\right)\right\|: w \in \operatorname{Inv}(\mathcal{A})\right\} \\
& =\inf \left\{\left\|\Phi(w) b \Phi(w)^{-1}\right\|: w \in \operatorname{Inv}(\mathcal{A})\right\} \\
& \geq \inf \left\{\left\|z b z^{-1}\right\|: \quad z \in \operatorname{Inv}(\mathcal{B})\right\} \\
& =\rho_{\mathcal{B}}(b) .
\end{aligned}
$$

Therefore,

$$
\rho_{\mathcal{B}}(b) \leq \inf \left\{\rho_{\mathcal{A}}(a): a \in \mathcal{A}, \Phi(a)=b\right\} .
$$

Now, let $\epsilon>0$ and choose $z \in \operatorname{Inv}(\mathcal{B})$ such that

$$
\left\|z b z^{-1}\right\| \leq \rho_{\mathcal{B}}(b)+\frac{\epsilon}{2}
$$

Since $z b z^{-1} \in \mathcal{B}$, according to the hypothesis, for any $\epsilon>0$, there exists $d \in \mathcal{A}$ such that

$$
\Phi(d)=z b z^{-1} \text { and }\|d\| \leq\left\|z b z^{-1}\right\|+\frac{\epsilon}{2} .
$$

Since $\Phi(\operatorname{Inv}(\mathcal{A})) \supseteq \operatorname{Inv}(\mathcal{B})$, we find $w \in \operatorname{Inv}(\mathcal{A})$ such that $\Phi(w)=z$. Notice that $y:=w^{-1} d w \in \mathcal{A}$ and

$$
\Phi(y)=\Phi(w)^{-1} \Phi(d) \Phi(w)=z^{-1}\left(z b z^{-1}\right) z=b .
$$

Now, using (2.2) and (2.3), we infer that

$$
\rho_{\mathcal{A}}(y) \leq\left\|w y w^{-1}\right\|=\|d\| \leq\left\|z b z^{-1}\right\|+\frac{\epsilon}{2} \leq \rho_{\mathcal{A}}(b)+\epsilon
$$

Therefore,

$$
\rho_{\mathcal{B}}(b) \geq \inf \left\{\rho_{\mathcal{A}}(a): a \in \mathcal{A}, \phi(a)=b\right\} .
$$

Using relation (2.1), it is easy to see that if $b \in \mathcal{B}$, then $\rho_{\mathcal{B}}(b)<1$ if and only if there exists $a \in \mathcal{A}$ such that $\Phi(a)=b$ and $\rho_{\mathcal{A}}(a)<1$. This completes the proof.

Corollary 2.2. Let $\mathcal{A}, \mathcal{B}$ be unital Banach algebras such that the group $\operatorname{Inv}(\mathcal{B})$ is connected. Let $\Phi: \mathcal{A} \rightarrow \mathcal{B}$ be a unital contractive homomorphism which is also a quotient interpolant. Then $\Phi$ is a spectral interpolant.

Proof. Let us prove that

$$
\Phi(\operatorname{Inv}(\mathcal{A}))=\operatorname{Inv}(\mathcal{B})
$$

The inclusion $\Phi(\operatorname{Inv}(\mathcal{A})) \subseteq \operatorname{Inv}(\mathcal{B})$ is clear. Conversely, let $x \in \operatorname{Inv}(\mathcal{B})$. Since $\operatorname{Inv}(\mathcal{B})$ is connected, it is well known that

$$
x=\exp \left(z_{1}\right) \cdots \exp \left(z_{k}\right)
$$


for some $z_{1}, \ldots, z_{k} \in \mathcal{B}$. Due to the hypothesis, there exist $w_{1}, \ldots, w_{k} \in \mathcal{A}$ such that $\Phi\left(w_{i}\right)=z_{i}, i=1, \ldots, k$. Denote $y:=\exp \left(w_{1}\right) \cdots \exp \left(w_{k}\right) \in \operatorname{Inv}(\mathcal{A})$ and notice that $\Phi(y)=\exp \left(\Phi\left(w_{1}\right)\right) \cdots \exp \left(\Phi\left(w_{k}\right)\right)=x$. Hence $\Phi(\operatorname{Inv}(\mathcal{A})) \supseteq \operatorname{Inv}(\mathcal{B})$ and (2.4) holds.

Remark 2.3. If $\mathcal{B}$ is a finite dimensional algebra, then $\operatorname{Inv}(\mathcal{B})=\exp (\mathcal{B})$, hence $\operatorname{Inv}(\mathcal{B})$ is connected.

Corollary 2.4. Let $\mathcal{A}$ be a unital Banach algebra and let $J$ be a closed two-sided ideal of $\mathcal{A}$. If any invertible element of $\mathcal{A} / J$ has an invertible lifting in $\mathcal{A}$, then the quotient homomorphism $\Phi: \mathcal{A} \rightarrow \mathcal{A} / J$ is a spectral interpolant, i.e., $\rho_{\mathcal{A} / J}(a+J)<1$ if and only if there exists $b \in a+J$ such that $\rho_{\mathcal{A}}(b)<1$.

Proof. Apply Theorem 2.1 to the quotient homomorphism $\Phi$.

Let us remark that, in general, there are invertible elements in $\mathcal{A} / J$ which can not be lifted to invertible elements in $\mathcal{A}$. For example, if $\pi: B\left(H^{2}\right) \rightarrow B\left(H^{2}\right) / K\left(H^{2}\right)$ is the quotient homomorphism into the Calkin algebra, and $S$ is the unilateral shift on the Hardy space $H^{2}$, then $\pi(S)$ is invertible and there is no invertible operator $T \in B\left(H^{2}\right)$ such that $\pi(T)=\pi(S)$.

An important particular case, when Corollary 2.4 can be applied, is when the quotient algebra $A / J$ is finite dimensional. Applications of this result will be considered in the next section.

\section{NONCOMMUTATIVE SPECTRAL COMMUTANT LIFTING AND INTERPOLATION}

Let $\mathbb{F}_{n}^{+}$be the unital free semigroup on $n$ generators $s_{1}, \ldots, s_{n}$, and let $e$ be its neutral element. For any $\sigma:=s_{i_{1}} \cdots s_{i_{k}} \in \mathbb{F}_{n}^{+}$we define its length $|\sigma|:=k$, and $|e|=0$. On the other hand, if $T_{i} \in B(\mathcal{H}), i=1, \ldots, n$, we denote $T_{\sigma}:=T_{i_{1}} \cdots T_{i_{k}}$ and $T_{e}:=I_{\mathcal{H}}$.

Let us recall from [Po1], [Po2], and [Po4] some results concerning the noncommutative dilation theory for $n$-tuples of operators. A sequence of operators $\mathcal{T}:=\left[T_{1}, \ldots, T_{n}\right], T_{i} \in B(\mathcal{H}), i=1, \ldots, n$, is called contractive (or row contraction) if $T_{1} T_{1}^{*}+\cdots+T_{n} T_{n}^{*} \leq I_{\mathcal{H}}$. We say that a sequence of isometries $\mathcal{V}:=\left[V_{1}, \ldots, V_{n}\right]$ on a Hilbert space $\mathcal{K} \supseteq \mathcal{H}$ is a minimal isometric dilation of $\mathcal{T}$ if the following properties are satisfied:

(i) $V_{1} V_{1}^{*}+\cdots+V_{n} V_{n}^{*} \leq I_{\mathcal{K}}$;

(ii) $\left.V_{i}^{*}\right|_{\mathcal{H}}=T_{i}^{*}, i=1, \ldots, n$;

(iii) $\mathcal{K}=\bigvee_{\alpha \in \mathbb{F}_{n}^{+}} V_{\alpha} \mathcal{H}$.

The minimal isometric dilation of $\mathcal{T}$ is uniquely determined up to an isomorphism. We need to recall the noncommutative commutant lifting theorem [P04] (see [SzF1, [SzF2], [DMP] for the classical case).

Let $\mathcal{T}:=\left[T_{1}, \ldots, T_{n}\right]$ be a contractive sequence of operators on a Hilbert space $\mathcal{H}$ and let $\mathcal{V}:=\left[V_{1}, \ldots, V_{n}\right]$ be its minimal isometric dilation on a Hilbert $\mathcal{K} \supseteq \mathcal{H}$. If $X \in B(\mathcal{H})$ and $X T_{i}=T_{i} X$ for any $i=1, \ldots, n$, then there exists $X_{\infty} \in B(\mathcal{K})$ satisfying the following properties:

(i) $X_{\infty} V_{i}=V_{i} X_{\infty}$, for any $i=1, \ldots, n$;

(ii) $\left.X_{\infty}^{*}\right|_{\mathcal{H}}=X^{*}$;

(iii) $\left\|X_{\infty}\right\|=\|X\|$. 
Let $\mathcal{T}:=\left[T_{1}, \ldots, T_{n}\right]$ be a row contraction with $T_{i} \in B(\mathcal{H})$ and let $\mathcal{V}:=$ $\left[V_{1}, \ldots, V_{n}\right]$ be its minimal isometric dilation on a Hilbert space $\mathcal{K} \supseteq \mathcal{H}$. Let $X \in\left\{T_{1}, \ldots, T_{n}\right\}^{\prime}$, and denote

$$
\operatorname{Dil}(X):=\left\{Y \in\left\{V_{1}, \ldots, V_{n}\right\}^{\prime}: P_{\mathcal{H}} Y=X P_{\mathcal{H}}\right\},
$$

where $P_{\mathcal{H}}$ is the orthogonal projection on $\mathcal{H}$. According to the noncommutative commutant lifting, we have $\operatorname{Dil}(X) \neq \emptyset$.

In what follows we obtain a noncommutative multivariable analogue of the spectral commutant lifting theorem of Bercovici-Foiaş-Tannenbaum [BFT].

Theorem 3.1. Let $\mathcal{T}:=\left[T_{1}, \ldots, T_{n}\right]$ be a contractive sequence of operators on a Hilbert space $\mathcal{H}$ and let $\mathcal{V}:=\left[V_{1}, \ldots, V_{n}\right]$ be its minimal isometric dilation on a Hilbert space $\mathcal{K} \supseteq \mathcal{H}$. If $\mathcal{H}$ is finite dimensional and $\mathcal{K} \ominus \mathcal{H}$ is hyperinvariant for $\left\{V_{1}, \ldots, V_{n}\right\}$, then

$$
\rho_{\left\{T_{1}, \ldots, T_{n}\right\}^{\prime}}(X)=\inf \left\{\rho_{\left\{V_{1}, \ldots, V_{n}\right\}^{\prime}}(Y): Y \in \operatorname{Dil}(X)\right\}
$$

for any $X \in\left\{T_{1}, \ldots, T_{n}\right\}^{\prime}$.

Proof. Let $\Phi:\left\{V_{1}, \ldots, V_{n}\right\}^{\prime} \rightarrow\left\{T_{1}, \ldots, T_{n}\right\}^{\prime}$ be defined by $\Phi(Y):=\left.P_{\mathcal{H}} Y\right|_{\mathcal{H}}$. Since $\mathcal{K} \ominus \mathcal{H}$ is hyperinvariant for $\left\{V_{1}, \ldots, V_{n}\right\}$, we have $Y^{*}(\mathcal{H}) \subseteq \mathcal{H}$ for any $Y \in\left\{V_{1}, \ldots, V_{n}\right\}^{\prime}$. Since $\mathcal{V}:=\left[V_{1}, \ldots, V_{n}\right]$ is the minimal isometric dilation of $\mathcal{T}$, we have $\left.V_{i}^{*}\right|_{\mathcal{H}}=T_{i}^{*}, i=1, \ldots, n$. Now, it is easy to see that

$$
\left(\left.P_{\mathcal{H}} Y\right|_{\mathcal{H}}\right) T_{i}=T_{i}\left(\left.P_{\mathcal{H}} Y\right|_{\mathcal{H}}\right) \quad \text { for any } i=1,2, \ldots, n \text {. }
$$

Therefore, the mapping $\Phi$ is well-defined. On the other hand, since $\mathcal{K} \ominus \mathcal{H}$ is hyperinvariant for $\left\{V_{1}, \ldots, V_{n}\right\}$, we infer that $\Phi$ is a unital contractive homomorphism, and $\Phi(Y)=X$ is equivalent to $P_{\mathcal{H}} Y=X P_{\mathcal{H}}$. According to the noncommutative commutant lifting theorem, for any $X \in\left\{T_{1}, \ldots, T_{n}\right\}^{\prime}$ there exists $Y \in\left\{V_{1}, \ldots, V_{n}\right\}^{\prime}$ such that $P_{\mathcal{H}} Y=X P_{\mathcal{H}}$ and $\|Y\|=\|X\|$. Therefore, $\Phi$ is a norm preserving interpolant. Since $\mathcal{H}$ is finite dimensional, the algebra $\left\{T_{1}, \ldots, T_{n}\right\}^{\prime}$ is finite dimensional. Applying Theorem 2.1 and Remark 2.3, in the particular case when $\mathcal{A}:=\left\{V_{1}, \ldots, V_{n}\right\}^{\prime}$ and $\mathcal{B}:=\left\{T_{1}, \ldots, T_{n}\right\}^{\prime}$, the result follows.

Corollary 3.2. Let $\mathcal{T}:=\left[T_{1}, \ldots, T_{n}\right]$ be a contractive sequence of operators on a Hilbert space $\mathcal{H}$ and let $\mathcal{V}:=\left[V_{1}, \ldots, V_{n}\right]$ be its minimal isometric dilation on a Hilbert space $\mathcal{K} \supseteq \mathcal{H}$. If $\mathcal{H}$ is finite dimensional and $\mathcal{K} \ominus \mathcal{H}$ is hyperinvariant for $\left\{V_{1}, \ldots, V_{n}\right\}$, then, given $X \in\left\{T_{1}, \ldots, T_{n}\right\}^{\prime}, \rho_{\left\{T_{1}, \ldots, T_{n}\right\}^{\prime}}(X)<1$ if and only if there exists $Y \in \operatorname{Dil}(X)$ such that $\rho_{\left\{V_{1}, \ldots, V_{n}\right\}^{\prime}}(Y)<1$.

In what follows, we use the noncommutative spectral commutant lifting theorem to obtain spectral versions of Sarason, Nevanlinna-Pick, and Carathéodory type interpolation for $F_{n}^{\infty} \bar{\otimes} B(\mathcal{K})$, the WOT-closed algebra generated by the spatial tensor product of the noncommutative analytic Toeplitz algebra $F_{n}^{\infty}$ and $B(\mathcal{K})$. In particular, we obtain interpolation results for matrix-valued analytic functions on the open unit ball of $\mathbb{C}^{n}$, in which one bounds the spectral radius of the interpolant.

According to Theorem 1.2 from [Po6], the commutant of $F_{n}^{\infty}$, which we denote by $R_{n}^{\infty}$, is equal to $U^{*} F_{n}^{\infty} U$, where $U$ is the unitary operator on $F^{2}\left(H_{n}\right)$ defined by $U\left(e_{i_{1}} \otimes e_{i_{2}} \otimes \cdots \otimes e_{i_{k}}\right)=e_{i_{k}} \otimes \cdots \otimes e_{i_{2}} \otimes e_{i_{1}}$. Moreover, the commutant of $R_{n}^{\infty}$ is equal to $F_{n}^{\infty}$.

A complete description of the invariant subspace structure of $F_{n}^{\infty}$ was obtained in [Po2, Theorem 2.2] (even in a more general setting). A subspace $\mathcal{N}$ of $F^{2}\left(H_{n}\right)$ is invariant under $S_{1}, \ldots, S_{n}$ if and only if $\mathcal{N}=\bigoplus_{\lambda \in \Lambda} U^{*} \varphi_{\lambda} U\left[F^{2}\left(H_{n}\right)\right]$, for some 
family $\left\{\varphi_{\lambda} \in F_{n}^{\infty}: \lambda \in \Lambda\right\}$ of isometries with orthogonal ranges (see also [Po6] and DP1]). Let us remark that $\mathcal{M} \subseteq F^{2}\left(H_{n}\right)$ is hyperinvariant for $\left\{S_{1}, \ldots, S_{n}\right\}$, i.e., invariant for $\left\{S_{1}, \ldots, S_{n}\right\}^{\prime}$, if and only if $U \mathcal{M}$ is invariant for $\left\{S_{1}, \ldots, S_{n}\right\}$.

Theorem 3.3. Let $\mathcal{K}$ be a finite dimensional Hilbert space and let $\mathcal{N} \subseteq F^{2}\left(H_{n}\right)$ be a finite dimensional subspace with the property that $\mathcal{N}$ and $U \mathcal{N}$ are invariant under $S_{1}^{*}, \ldots, S_{n}^{*}$. Then $X \in B(\mathcal{N} \otimes \mathcal{K})$ commutes with each $\left.P_{\mathcal{N}} S_{i}\right|_{\mathcal{N}} \otimes I_{\mathcal{K}}, i=1, \ldots, n$, and

$$
\rho_{\left.P_{\mathcal{N}} R_{n}^{\infty}\right|_{\mathcal{N}} \bar{\otimes} B(\mathcal{K})}(X)<1
$$

if and only there exists $\Psi \in R_{n}^{\infty} \bar{\otimes} B(\mathcal{K})$ such that

$$
P_{\mathcal{N} \otimes \mathcal{K}} \Psi=X P_{\mathcal{N} \otimes \mathcal{K}} \quad \text { and } \rho_{R_{n}^{\infty} \bar{\otimes} B(\mathcal{K})}(\Psi)<1 .
$$

Proof. According to $\mathrm{Po} 8$, we have

$$
\mathcal{B}:=\left\{\left.P_{\mathcal{N}} S_{i}\right|_{\mathcal{N}} \otimes I_{\mathcal{K}}, i=1, \ldots, n\right\}^{\prime}=\left.P_{\mathcal{N} \otimes \mathcal{K}}\left(R_{n}^{\infty} \bar{\otimes} B(\mathcal{K})\right)\right|_{\mathcal{N} \otimes \mathcal{K}}
$$

Notice that $\mathcal{B}$ is a finite dimensional algebra. Let $\mathcal{A}:=R_{n}^{\infty} \bar{\otimes} B(\mathcal{K})$ and define $\Phi: \mathcal{A} \rightarrow \mathcal{B}$ by $\Phi(Y)=\left.P_{\mathcal{N} \otimes \mathcal{K}} Y\right|_{\mathcal{N} \otimes \mathcal{K}}$. Since $S_{i}^{*}(U \mathcal{N}) \subseteq U \mathcal{N}$ for any $i=1, \ldots, n$, and $\left\{S_{1} \otimes I_{\mathcal{K}}, \ldots, S_{n} \otimes I_{\mathcal{K}}\right\}^{\prime}=R_{n}^{\infty} \bar{\otimes} B(\mathcal{K})$, it is easy to see that $\left[F^{2}\left(H_{n}\right) \otimes \mathcal{K}\right] \ominus$ $[\mathcal{N} \otimes \mathcal{K}]$ is hyperinvariant for $\left\{S_{1} \otimes I_{\mathcal{K}}, \ldots, S_{n} \otimes I_{\mathcal{K}}\right\}$ and the mapping $\Phi$ is a unital contractive homomorphism. Since $\mathcal{N}$ is invariant under $S_{1}^{*}, \ldots, S_{n}^{*}$, it is clear that the operator matrix $\left[\left.P_{\mathcal{N}} S_{1}\right|_{\mathcal{N}}, \ldots,\left.P_{\mathcal{N}} S_{n}\right|_{\mathcal{N}}\right]$ is a $C_{0}$-row contraction and its minimal isometric dilation is $\left[S_{1}, \ldots, S_{n}\right]$ (see [Po1]). Therefore, the minimal isometric dilation of $\left[\left.P_{\mathcal{N}} S_{1}\right|_{\mathcal{N}} \otimes I_{\mathcal{K}}, \ldots,\left.P_{\mathcal{N}} S_{n}\right|_{\mathcal{N}} \otimes I_{\mathcal{K}}\right]$ is $\left[S_{1} \otimes I_{\mathcal{K}}, \ldots, S_{n} \otimes I_{\mathcal{K}}\right]$. According to the noncommutative commutant lifting theorem, for any $X \in \mathcal{B}$ there exists $\Psi \in R_{n}^{\infty} \bar{\otimes} B(\mathcal{K})$, such that $P_{\mathcal{N} \otimes \mathcal{K}} \Psi=X P_{\mathcal{N} \otimes \mathcal{K}}$ and $\|X\|=\|\Psi\|$. Therefore, $\Phi(\Psi)=X$ and $\Phi$ is a norm preserving interpolant. Applying Corollary 3.2, the result follows.

Notice that the element $\Psi$ in Theorem 3.3 satisfies $\|\Psi\|_{\mathrm{sp}} \leq \rho_{R_{n}^{\infty} \bar{\otimes} B(\mathcal{K})}(\Psi)<1$. It would be nice to know if $\rho_{R_{n}^{\infty} \bar{\otimes} B(\mathcal{K})}(\Psi)=\|\Psi\|_{\text {sp }}$ for any $\Psi \in R_{n}^{\infty} \bar{\otimes} B(\mathcal{K})$. This equality holds if $n=1$ (see $[\overline{\mathrm{BFT}}]$ ).

Let us remark that the finite dimensionality hypothesis can be dropped in Theorem 3.3 for those subspaces $\mathcal{N}$ and $\mathcal{K}$ for which one can prove that any invertible element $\left.f \in P_{\mathcal{N}} R_{n}^{\infty}\right|_{\mathcal{N}} \bar{\otimes} B(\mathcal{K})$ can be lifted to an invertible element $g \in R_{n}^{\infty} \bar{\otimes} B(\mathcal{K})$, i.e., $\left.P_{\mathcal{N} \otimes \mathcal{K}} g\right|_{\mathcal{N} \otimes \mathcal{K}}=f$. We do not have yet any nontrivial example when this lifting property holds and $\mathcal{N}, \mathcal{K}$ are infinite dimensional.

Let $J$ be a WOT-closed, two-sided ideal of $F_{n}^{\infty}$ and define $J(1):=\{\Psi(1): \Psi \in J\}$ and $\left.\mathcal{N}_{J}:=F^{2}\left(H_{n}\right) \ominus J(1)\right\}$. Let us remark that $\mathcal{N}_{J}$ and $U \mathcal{N}_{J}$ are invariant subspaces under $S_{i}^{*}, i=1, \ldots, n$, therefore, Theorem 3.3 works in the case when $\operatorname{dim} \mathcal{N}_{J}<\infty$.

Corollary 3.4. Let $\mathcal{K}$ be a finite dimensional Hilbert space and let $J$ be a WOTclosed two-sided ideal of $F_{n}^{\infty}$ such that $\operatorname{dim} \mathcal{N}_{J}<\infty$. Then the quotient homomorphism

$$
\Phi: F_{n}^{\infty} \bar{\otimes} B(\mathcal{K}) \rightarrow F_{n}^{\infty} \bar{\otimes} B(\mathcal{K}) /(J \bar{\otimes} B(\mathcal{K}))
$$

is a spectral interpolant.

Proof. According to $\underline{\mathrm{ArPo} 2}$, the quotient algebra $F_{n}^{\infty} \bar{\otimes} B(\mathcal{K}) /(J \bar{\otimes} B(\mathcal{K}))$ is completely isometrically isomorphic to $\left.P_{\mathcal{N}_{J}} F_{n}^{\infty}\right|_{\mathcal{N}_{J}} \bar{\otimes} B(\mathcal{K})$, which is finite dimensional. Using Theorem 3.3, we infer that $\Phi$ is a spectral interpolant. The proof is complete. 
It will be interesting to see if this result remains true if $\mathcal{N}_{J}$ is infinite dimensional (at least for some particular cases, if not in general). The obstruction in the infinite dimensional case seems to be the lifting of the invertible elements of a quotient algebra $\mathcal{A} / J$ to invertible elements of $\mathcal{A}$ (see Section 2 for an example). In the finite dimensional case, Corollary 3.4 leads to our spectral interpolation results for $F_{n}^{\infty}$ (see Theorem 3.6 and Theorem 3.8).

Let $F_{s}^{2}\left(H_{n}\right)$ be the symmetric Fock space and $\mathcal{W}_{n}^{\infty}$ be the WOT-closed algebra generated by $B_{i}:=\left.P_{F_{s}^{2}\left(H_{n}\right)} S_{i}\right|_{F_{s}^{2}\left(H_{n}\right)}, i=1, \ldots, n$, and the identity. This algebra has been studied in [Po9], Arv], ArPo2, [DP3]. The following theorem can be seen as a spectral version of Sarason's interpolation theorem for $H^{\infty}(\mathbb{D})$ (see $[\underline{\mathrm{S}}$ ), in a commutative and multivariable setting.

Theorem 3.5. Let $\mathcal{E} \subseteq F_{s}^{2}\left(H_{n}\right)$ be a finite dimensional invariant subspace under $B_{1}^{*}, \ldots, B_{n}^{*}$ and let $\mathcal{K}$ be a finite dimensional Hilbert space. Then $f \in B(\mathcal{E} \otimes \mathcal{K})$ commutes with each $\left.P_{\mathcal{E}} B_{i}\right|_{\mathcal{E}} \otimes I_{\mathcal{K}}, i=1, \ldots, n$, and

$$
\left.\rho_{P \mathcal{E} \otimes \mathcal{K}}\left(\mathcal{W}_{n}^{\infty} \bar{\otimes} B(\mathcal{K})\right)\right|_{\mathcal{E} \otimes \mathcal{K}}(f)<1
$$

if and only if there exists $g \in \mathcal{W}_{n}^{\infty} \bar{\otimes} B(\mathcal{K})$ such that

$$
\left.P_{\mathcal{E} \otimes \mathcal{K}} g\right|_{\mathcal{E} \otimes \mathcal{K}}=f \text { and } \rho_{\mathcal{W}_{n}^{\infty} \bar{\otimes} B(\mathcal{K})}(g)<1 .
$$

Proof. Since $F_{s}^{2}\left(H_{n}\right)$ is invariant under each $S_{i}^{*}, i=1, \ldots, n$, it is easy to see that $\mathcal{E}$ has the same property. Taking into account that $\mathcal{W}_{n}^{\infty}$ is the compression of $F_{n}^{\infty}$ to the symmetric Fock space, one can see that $f$ commutes with $\left.P_{\mathcal{E} \otimes \mathcal{K}}\left(S_{i} \otimes I_{\mathcal{K}}\right)\right|_{\mathcal{E} \otimes \mathcal{K}}$. As in the proof of Theorem 3.3, using the noncommutative commutant lifting theorem, we find $\phi \in F_{n}^{\infty} \bar{\otimes} B(\mathcal{K})$ such that $\left.P_{\mathcal{E} \otimes \mathcal{K}}\left(U^{*} \otimes I_{\mathcal{K}}\right) \phi(U \otimes I)\right|_{\mathcal{E} \otimes \mathcal{K}}=f$ and $\|f\|=\|\phi\|$. Hence, $\left.P_{\mathcal{E} \otimes \mathcal{K}} \phi\right|_{\mathcal{E} \otimes \mathcal{K}}=f$. Setting $g:=\left.P_{F_{s}^{2}\left(H_{n}\right) \otimes \mathcal{K}} \phi\right|_{F_{s}^{2}\left(H_{n}\right) \otimes \mathcal{K}} \in \mathcal{W}_{n}^{\infty} \bar{\otimes} B(\mathcal{K})$, we have $\left.P_{\mathcal{E} \otimes \mathcal{K}} g\right|_{\mathcal{E} \otimes \mathcal{K}}=f$ and $\|f\| \leq\|g\| \leq\|\phi\|=\|f\|$. This shows that $\|f\|=\|g\|$. Define $\mathcal{A}:=\mathcal{W}_{n}^{\infty} \bar{\otimes} B(\mathcal{K}), \quad \mathcal{B}:=\left.P_{\mathcal{E} \otimes \mathcal{K}}\left(\mathcal{W}_{n}^{\infty} \bar{\otimes} B(\mathcal{K})\right)\right|_{\mathcal{E} \otimes \mathcal{K}}$ and let $\Phi: \mathcal{A} \rightarrow \mathcal{B}$ be defined by $\Phi(g):=P_{\mathcal{E} \otimes \mathcal{K}}(g)_{\mathcal{E} \otimes \mathcal{K}}$. We just proved that $\Phi$ is a unital contractive homomorphism and also a norm preserving interpolant. Now, the result follows by applying the results of Section 2 in our setting.

Let us remark that a result similar to Corollary 3.4 holds for the algebra $\mathcal{W}_{n}^{\infty} \bar{\otimes}$ $B(\mathcal{K})$.

In what follows we obtain a spectral version of Nevanlinna-Pick interpolation for the noncommutative analytic Toeplitz algebra $F_{n}^{\infty}$ (see [ArPo2, [DP3], and [Po8]). As mentioned in the first section, there exists a unital contractive homomorphism

$$
\Psi: F_{n}^{\infty} \bar{\otimes} B(\mathcal{K}) \rightarrow H^{\infty}\left(\mathbb{B}_{n}\right) \bar{\otimes} B(\mathcal{K})
$$

defined by $[\Psi(f)](\lambda):=f(\lambda), \lambda \in \mathbb{B}_{n}$.

Theorem 3.6. Let $\mathcal{K}$ be a finite dimensional Hilbert space, $W_{j} \in B(\mathcal{K})$, and $\lambda_{j}, j=1, \ldots, k$, be distinct elements in $\mathbb{B}_{n}$. Then there exists $\Phi \in F_{n}^{\infty} \bar{\otimes} B(\mathcal{K})$ such that

$$
\rho_{F_{n}^{\infty} \bar{\otimes} B(\mathcal{K})}(\Phi)<1 \quad \text { and } \quad \Phi\left(\lambda_{j}\right)=W_{j}, j=1, \ldots, k,
$$

if and only if there exist invertible operators $M_{j} \in B(\mathcal{K}), j=1, \ldots, k$, such that

$$
\left[\frac{I_{\mathcal{K}}-\left(M_{i} W_{i} M_{i}^{-1}\right)\left(M_{j} W_{j} M_{j}^{-1}\right)^{*}}{1-\left\langle\lambda_{i}, \lambda_{j}\right\rangle}\right]_{1 \leq i, j \leq k}>0 .
$$


Proof. Let $\lambda_{j}:=\left(\lambda_{j 1}, \ldots, \lambda_{j n}\right) \in \mathbb{B}_{n}, j=1, \ldots, k$. For any $\alpha:=s_{j_{1}} s_{j_{2}} \ldots s_{j_{m}}$ in $\mathbb{F}_{n}^{+}$, let $\lambda_{j \alpha}:=\lambda_{j j_{1}} \lambda_{j j_{2}} \ldots \lambda_{j j_{m}}$ and $\lambda_{e}:=1$. Define $z_{\lambda_{j}} \in F^{2}\left(H_{n}\right)$ by setting

$$
z_{\lambda_{j}}:=\sum_{\alpha \in \mathbb{F}_{n}^{+}} \bar{\lambda}_{j \alpha} e_{\alpha}, \quad j=1,2, \ldots, k .
$$

Let $\mathcal{N}:=\operatorname{span}\left\{z_{\lambda_{j}}: j=1, \ldots, k\right\}$ and $X \in B(\mathcal{N} \otimes \mathcal{K})$ be defined by

$$
X^{*}\left(z_{\lambda_{j}} \otimes h\right):=z_{\lambda_{j}} \otimes W_{j}^{*} h, \quad h \in \mathcal{K} .
$$

Notice that $S_{i}^{*} z_{\lambda_{j}}=\bar{\lambda}_{j i} z_{\lambda_{j}}$ for any $i=1, \ldots, n ; j=1, \ldots, k$. Hence, the subspaces $\mathcal{N}$ and $U \mathcal{N}$ are invariant under each $S_{i}^{*}, i=1, \ldots, n$. Define $T_{i} \in$ $B(\mathcal{N} \otimes \mathcal{K})$ by $T_{i}:=\left.P_{\mathcal{N}} S_{i}\right|_{\mathcal{N}} \otimes I_{\mathcal{K}}$. Since $z_{\lambda_{1}}, \ldots, z_{\lambda_{k}}$ are linearly independent, the operator $X \in B(\mathcal{N} \otimes \mathcal{K})$ given by (3.2) is well defined.

Notice that $X T_{i}=T_{i} X$ for any $i=1, \ldots, k$. Indeed,

$$
\begin{aligned}
T_{i}^{*} X^{*}\left(z_{\lambda_{j}} \otimes h\right) & =T_{i}^{*}\left(z_{\lambda_{j}} \otimes W_{j}^{*} h\right)=S_{i}^{*} z_{\lambda_{j}} \otimes W_{j}^{*} h \\
& =\bar{\lambda}_{j i} z_{\lambda_{j}} \otimes W_{j}^{*} h
\end{aligned}
$$

and

$$
X^{*} T_{i}^{*}\left(z_{\lambda_{j}} \otimes h\right)=X^{*}\left(\bar{\lambda}_{j i} z_{\lambda_{j}} \otimes h\right)=\bar{\lambda}_{j i} z_{\lambda_{j}} \otimes W_{j}^{*} h .
$$

Applying Theorem 3.3, we infer that

$$
\rho_{\left\{T_{1}, \ldots, T_{n}\right\}^{\prime}}(X)<1
$$

if and only there exists $\Phi \in F_{n}^{\infty} \bar{\otimes} B(\mathcal{K})$ such that

$$
P_{\mathcal{N} \otimes \mathcal{K}}\left(U^{*} \otimes I\right) \Phi(U \otimes I)=X P_{\mathcal{N} \otimes \mathcal{K}} \quad \text { and } \quad \rho_{F_{n}^{\infty} \bar{\otimes} B(\mathcal{K})}(\Phi)<1 .
$$

Since $\left[F^{2}\left(H_{n}\right) \otimes \mathcal{K}\right] \ominus[\mathcal{N} \otimes \mathcal{K}]$ is hyperinvariant for $\left\{S_{1} \otimes I_{\mathcal{K}}, \ldots, S_{n} \otimes I_{\mathcal{K}}\right\}$, the first relation in (3.4) is equivalent to

$$
\left.P_{\mathcal{N} \otimes \mathcal{K}}\left(U^{*} \otimes I\right) \Phi(U \otimes I)\right|_{\mathcal{N} \otimes \mathcal{K}}=X .
$$

Since $U\left(z_{\lambda_{j}}\right)=z_{\lambda_{j}}, j=1, \ldots, k$, and $\left\langle\phi, z_{\lambda_{i}}\right\rangle=\phi\left(\lambda_{i}\right)$ for any $\phi:=\sum_{\alpha \in \mathbb{F}_{n}^{+}} a_{\alpha} e_{\alpha}$ in $F^{2}\left(H_{n}\right)$, it is easy to see that

$$
\begin{aligned}
& \left\langle\left(U^{*} \otimes I\right) \Phi(U \otimes I)\left(z_{\lambda_{j}} \otimes h\right), z_{\lambda_{j}} \otimes h^{\prime}\right\rangle \\
& =\left\langle z_{\lambda_{j}}, z_{\lambda_{j}}\right\rangle\left\langle\Phi\left(\lambda_{j}\right) h, h^{\prime}\right\rangle=\left\langle X\left(z_{\lambda_{j}} \otimes h\right), z_{\lambda_{j}} \otimes h^{\prime}\right\rangle \\
& =\left\langle\Phi\left(z_{\lambda_{j}} \otimes h\right), z_{\lambda_{j}} \otimes h^{\prime}\right\rangle=\left\langle z_{\lambda_{j}}, z_{\lambda_{j}}\right\rangle\left\langle W_{j} h, h^{\prime}\right\rangle .
\end{aligned}
$$

for any $j=1, \ldots, k$, and $h, h^{\prime} \in \mathcal{K}$. This shows that (3.5) holds if and only if $\Phi\left(\lambda_{j}\right)=W_{j}$ for any $j=1, \ldots, k$. Notice that relation (3.3) holds if and only if there exists $M \in \operatorname{Inv}\left(\left\{T_{1}, \ldots, T_{n}\right\}^{\prime}\right)$ such that $\left\|M X M^{-1}\right\|<1$. It is easy to see that $M^{*}\left(z_{\lambda_{j}} \otimes h\right)=z_{\lambda_{j}} \otimes M_{j}^{*} h, h \in \mathcal{K}$, for some invertible operators $M_{j} \in B(\mathcal{K}), j=$ $1, \ldots, k$. On the other hand, notice that

$$
M^{*-1} X^{*} M^{*}\left(z_{\lambda_{j}} \otimes h\right)=z_{\lambda_{j}} \otimes\left(M_{j} W_{j} M_{j}^{-1}\right)^{*} h
$$

and $\left\|M X M^{-1}\right\|<1$ is equivalent to $I_{\mathcal{N} \otimes \mathcal{K}}-\left(M X M^{-1}\right)\left(M X M^{-1}\right)^{*}>0$, which is equivalent to (3.1). This completes the proof.

Let us remark that the inequality (3.1) can be replaced with

$$
\left.\rho_{P_{\mathcal{N}} F_{n}^{\infty}}\right|_{\mathcal{N}} \bar{\otimes} B(\mathcal{K})(X)<1 .
$$


In the particular case when $n=1$, we find again Theorem 4 from BFT. As mentioned in $\overline{\mathrm{BFT}}$, since $\left.P_{\mathcal{N}} F_{n}^{\infty}\right|_{\mathcal{N}} \bar{\otimes} B(\mathcal{K})$ is finite dimensional, conditions of type (3.6) can be checked using computer algorithms.

Corollary 3.7. Let $\mathcal{K}$ be a finite dimensional Hilbert space, $W_{j} \in B(\mathcal{K})$, and $\lambda_{j}, j=1, \ldots, k$, be distinct elements in $\mathbb{B}_{n}$. If there exist invertible operators $M_{j} \in B(\mathcal{K}), j=1, \ldots, k$, such that

$$
\left[\frac{I_{\mathcal{K}}-\left(M_{i} W_{i} M_{i}^{-1}\right)\left(M_{j} W_{j} M_{j}^{-1}\right)^{*}}{1-\left\langle\lambda_{i}, \lambda_{j}\right\rangle}\right]_{1 \leq i, j \leq k}>0,
$$

then there exists $f \in H^{\infty}\left(\mathbb{B}_{n}\right) \bar{\otimes} B(\mathcal{K})$ such that

$$
f\left(\lambda_{j}\right)=W_{j}, j=1, \ldots, k, \quad \text { and } \quad \sup _{\lambda \in \mathbb{B}_{n}}\|f(\lambda)\|_{\mathrm{sp}}<1 .
$$

Proof. Using Theorem 3.6, we find $f \in F_{n}^{\infty} \bar{\otimes} B(\mathcal{K})$ such that $f\left(\lambda_{j}\right)=W_{j}, i=$ $1, \ldots, k$, and $\rho_{F_{n}^{\infty} \bar{\otimes} B(\mathcal{K})}(f)<1$. As in the proof of Theorem 2.1, we infer that

$$
\|\Psi(f)\|_{\mathrm{sp}} \leq \rho_{H^{\infty}\left(\mathbb{B}_{n}\right) \bar{\otimes} B(\mathcal{K})}(\Psi(f)) \leq \rho_{F_{n}^{\infty} \bar{\otimes} B(\mathcal{K})}(f)<1 .
$$

On the other hand, similarly to [BFT Proposition 3], one can prove that

$$
\|\Psi(f)\|_{\mathrm{sp}}=\sup _{\lambda \in \mathbb{B}_{n}}\|f(\lambda)\|_{\mathrm{sp}} .
$$

This completes the proof.

Let $\mathcal{P}_{m}$ be the set of all polynomials in $F^{2}\left(H_{n}\right)$ of degree $\leq m$, and let $\mathcal{P}_{m}^{\infty}:=$ $\left\{p\left(S_{1}, \ldots, S_{n}\right): p \in \mathcal{P}_{m}\right\}$. Let $J_{>m}^{\infty}$ be the WOT-closed two-sided ideal of $F_{n}^{\infty}$ generated by $\left\{S_{\alpha}: \alpha \in \mathbb{F}_{n}^{+},|\alpha|=m+1\right\}$. The following result is a spectral version of the noncommutative Carathéodory interpolation problem for $F_{n}^{\infty}$ (see [Po6] and [Po8]).

Theorem 3.8. Let $\mathcal{K}$ be a finite dimensional Hilbert space and let $p \in \mathcal{P}_{m}^{\infty} \bar{\otimes} B(\mathcal{K})$. Then there exists $\Phi \in F_{n}^{\infty} \bar{\otimes} B(\mathcal{K})$ with

$$
\rho_{F_{n}^{\infty} \bar{\otimes} B(\mathcal{K})}(\Phi)<1
$$

such that $\Phi=p+g$ for some $g \in J_{>m}^{\infty} \bar{\otimes} B(\mathcal{K})$ if and only if

$$
\rho_{\mathcal{C}}\left[\left.P_{\mathcal{P}_{m} \otimes \mathcal{K}}\left(U^{*} \otimes I\right) p(U \otimes I)\right|_{\mathcal{P}_{m} \otimes \mathcal{K}}\right]<1
$$

where $\mathcal{C}:=\left.P_{\mathcal{P}_{m} \otimes \mathcal{K}}\left(R_{n}^{\infty} \bar{\otimes} B(\mathcal{K})\right)\right|_{\mathcal{P}_{m} \otimes \mathcal{K}}$.

Proof. Let $\mathcal{N}:=\mathcal{P}_{m}$ and $X:=\left.P_{\mathcal{P}_{m} \otimes \mathcal{K}}\left(U^{*} \otimes I\right) p(U \otimes I)\right|_{\mathcal{P}_{m} \otimes \mathcal{K}}$. Notice that $X$ commutes with each $\left.P_{\mathcal{P}_{m}} S_{i}\right|_{\mathcal{P}_{m}} \otimes I_{\mathcal{K}}, i=1, \ldots, n$, and $\mathcal{P}_{m}=U \mathcal{P}_{m}$ is invariant under each $S_{1}^{*}, \ldots, S_{n}^{*}$. According to Theorem 3.3, relation (3.7) holds if and only if there exists $\Phi \in F_{n}^{\infty} \bar{\otimes} B(\mathcal{K})$ with $P_{\mathcal{P}_{m} \otimes \mathcal{K}}\left(U^{*} \otimes I\right) \Phi(U \otimes I)=X P_{\mathcal{P}_{m} \otimes \mathcal{K}}$ and $\rho_{F_{n}^{\infty} \bar{\otimes} B(\mathcal{K})}(\Phi)<1$. Hence, we infer that

$$
\left.P_{\mathcal{P}_{m} \otimes \mathcal{K}}\left(U^{*} \otimes I\right)(\Phi-p)(U \otimes I)\right|_{\mathcal{P}_{m} \otimes \mathcal{K}}=0 .
$$

On the other hand, every element $f \in F_{n}^{\infty} \bar{\otimes} B(\mathcal{K})$ has a unique Fourier expansion $f \sim \sum_{\alpha \in \mathbb{F}_{n}^{+}} S_{\alpha} \otimes W_{(\alpha)}$ determined by

$$
f(1 \otimes h)=\sum_{\alpha \in \mathbb{F}_{n}^{+}} e_{\alpha} \otimes W_{(\alpha)} h \in F^{2}\left(H_{n}\right) \otimes \mathcal{K},
$$


where $W_{(\alpha)} \in B(\mathcal{K})$ are given by $\left\langle W_{(\alpha)} h, k\right\rangle=\left\langle f(1 \otimes h), e_{\alpha} \otimes k\right\rangle$ for any $h, k \in \mathcal{K}$, and $\alpha \in \mathbb{F}_{n}^{+}$(see $\left.[\mathrm{Po} 8]\right)$. Using now relation (3.8), one can easily see that $g:=$ $\Phi-p \in J_{>m}^{\infty} \bar{\otimes} B(\mathcal{K})$. This completes the proof.

Using Theorem 3.5, one can obtain a version of Theorem 3.8 for the algebra $\mathcal{W}_{n}^{\infty} \bar{\otimes} B(\mathcal{K})$, in a similar manner. We leave this task to the reader.

\section{Spectral tangential Commutant lifting in SEVERAl Variables}

Let $\mathcal{T}:=\left[T_{1}, \ldots, T_{n}\right]$ be a row contraction with $T_{i} \in B(\mathcal{H})$, and $\mathcal{V}:=\left[V_{1}, \ldots, V_{n}\right]$ be its minimal isometric dilation on a Hilbert space $\mathcal{K} \supseteq \mathcal{H}$. Let $\mathcal{M} \subseteq \mathcal{H}$ be an invariant subspace under each $T_{i}^{*}, i=1, \ldots, n$, and $X \in B(\mathcal{H})$ be such that $X \mathcal{H} \subseteq \mathcal{M}$ and

$$
\left(\left.P_{\mathcal{M}} T_{i}\right|_{\mathcal{M}}\right) X=X T_{i}, \text { for any } i=1, \ldots, n .
$$

According to the noncommutative commutant lifting theorem, there exists $Y \in$ $\left\{V_{1}, \ldots, V_{n}\right\}^{\prime}$ with $P_{\mathcal{M}} Y=X P_{\mathcal{H}}$. Define

$$
\operatorname{Dil}_{\mathcal{M}}(X):=\left\{Y \in\left\{V_{1}, \ldots, V_{n}\right\}^{\prime}: P_{\mathcal{M}} Y=X P_{\mathcal{H}}\right\}
$$

and

$$
\rho_{\mathcal{M},\left\{T_{1}, \ldots, T_{n}\right\}^{\prime}}(X):=\inf \left\{\left\|P_{Z^{*} \mathcal{M}} Z^{-1} X Z\right\|: Z \in \operatorname{Inv}\left(\left\{T_{1}, \ldots, T_{n}\right\}^{\prime}\right)\right\} .
$$

Notice that if $\mathcal{M}=\mathcal{H}$, then $\rho_{\mathcal{M},\left\{T_{1}, \ldots, T_{n}\right\}^{\prime}}(X)=\rho_{\left\{T_{1}, \ldots, T_{n}\right\}^{\prime}}(X)$.

In what follows we extend the spectral tangential commutant lifting theorem of Bercovici and Foiaş $[\mathrm{BF}]$ to our noncommutative multivariable setting.

Theorem 4.1. Let $\mathcal{T}:=\left[T_{1}, \ldots, T_{n}\right]$ be a contractive sequence of operators on a Hilbert space $\mathcal{H}$ and let $\mathcal{V}:=\left[V_{1}, \ldots, V_{n}\right]$ be its minimal isometric dilation on a Hilbert space $\mathcal{K} \supseteq \mathcal{H}$. If $\mathcal{H}$ is finite dimensional, $\mathcal{K} \ominus \mathcal{H}$ is hyperinvariant for $\left\{V_{1}, \ldots, V_{n}\right\}$, and $\mathcal{M} \subseteq \mathcal{H}$ is an invariant subspace under each $T_{i}^{*}, i=1, \ldots, n$, then, for every $X \in B(\mathcal{H})$ such that $X \mathcal{H} \subseteq \mathcal{M}$ and $\left(\left.P_{\mathcal{M}} T_{i}\right|_{\mathcal{M}}\right) X=X T_{i}, \quad i=$ $1, \ldots, n$, we have

$$
\rho_{\mathcal{M},\left\{T_{1}, \ldots, T_{n}\right\}^{\prime}}(X)=\inf \left\{\rho_{\left\{V_{1}, \ldots, V_{n}\right\}^{\prime}}(Y): Y \in \operatorname{Dil}_{\mathcal{M}}(X)\right\} .
$$

Proof. Denote the right hand side of (4.2) by $t$. Let $\epsilon>0$ and choose $Y \in \operatorname{Dil}_{\mathcal{M}}(X)$ such that $\rho_{\left\{V_{1}, \ldots, V_{n}\right\}^{\prime}}(Y)<t+\epsilon$. Hence, there is $W \in \operatorname{Inv}\left(\left\{V_{1}, \ldots, V_{n}\right\}^{\prime}\right)$ such that $\left\|W^{-1} Y W\right\|<t+\epsilon$. Since $\mathcal{K} \ominus \mathcal{H}$ is hyperinvariant for $\left\{V_{1}, \ldots, V_{n}\right\}$, we infer that $P_{\mathcal{H}} W P_{\mathcal{H}}=P_{\mathcal{H}} W$. Let $Z:=\left.P_{\mathcal{H}} W\right|_{\mathcal{H}}$ and notice that $Z \in \operatorname{Inv}\left(\left\{T_{1}, \ldots, T_{n}\right\}^{\prime}\right)$ and

$$
Z^{-1}=\left.P_{\mathcal{H}} W^{-1}\right|_{\mathcal{H}}
$$

The subspace $\mathcal{M}_{*}:=Z^{*} \mathcal{M}$ is invariant under each $T_{i}^{*}, i=1, \ldots, n$, and satisfies $\mathcal{M}_{*}=\mathcal{H} \ominus Z^{-1}(\mathcal{H} \ominus \mathcal{M})$. Hence, we deduce the relations

$$
P_{\mathcal{M}_{*}} Z^{-1}=P_{\mathcal{M}_{*}} Z^{-1} P_{\mathcal{M}} \text { and } P_{\mathcal{M}} Z=P_{\mathcal{M}} Z P_{\mathcal{M}_{*}} .
$$

Since $Y \in \operatorname{Dil}_{\mathcal{M}}(X)$ and $\mathcal{K} \ominus \mathcal{H}$ is hyperinvariant for $\left\{V_{1}, \ldots, V_{n}\right\}$, we can use (4.4) and (4.3) to infer that

$$
\begin{aligned}
\left\|P_{\mathcal{M}_{*}} Z^{-1} X Z\right\| & =\left\|P_{\mathcal{M}_{*}} Z^{-1}\left(\left.P_{\mathcal{M}} Y\right|_{\mathcal{H}}\right) Z\right\|=\left\|P_{\mathcal{M}_{*}} Z^{-1}\left(\left.P_{\mathcal{H}} Y\right|_{\mathcal{H}}\right) Z\right\| \\
& =\left\|P_{\mathcal{M}_{*}}\left(\left.P_{\mathcal{H}} W^{-1}\right|_{\mathcal{H}}\right)\left(\left.P_{\mathcal{H}} Y\right|_{\mathcal{H}}\right)\left(\left.P_{\mathcal{H}} W\right|_{\mathcal{H}}\right)\right\| \leq\left\|\left.P_{\mathcal{H}}\left(W^{-1} Y W\right)\right|_{\mathcal{H}}\right\| \\
& \leq\left\|W^{-1} Y W\right\|<t+\epsilon .
\end{aligned}
$$

Since $\epsilon>0$, we deduce that $\rho_{\mathcal{M},\left\{T_{1}, \ldots, T_{n}\right\}^{\prime}}(X) \leq t$. 
Now, let us prove the converse. Let $\epsilon>0$ and choose $Z \in \operatorname{Inv}\left(\left\{T_{1}, \ldots, T_{n}\right\}^{\prime}\right)$ such that

$$
\left\|P_{\mathcal{M}_{*}} Z^{-1} X Z\right\| \leq \rho_{\mathcal{M},\left\{T_{1}, \ldots, T_{n}\right\}^{\prime}}(X)+\epsilon .
$$

Since $\left\{T_{1}, \ldots, T_{n}\right\}^{\prime}$ is finite dimensional, we use Theorem 2.1 and Remark 2.3 when $\Phi:\left\{V_{1}, \ldots, V_{n}\right\}^{\prime} \rightarrow\left\{T_{1}, \ldots, T_{n}\right\}^{\prime}$ and $\Phi(W)=\left.P_{\mathcal{H}} W\right|_{\mathcal{H}}$, to find $W \in$ $\operatorname{Inv}\left(\left\{V_{1}, \ldots, V_{n}\right\}^{\prime}\right)$ such that $Z=\left.P_{\mathcal{H}} W\right|_{\mathcal{H}}$. Denote $X_{*}:=P_{\mathcal{M}_{*}} Z^{-1} X Z$ and notice that

$$
\left(\left.P_{\mathcal{M}_{*}} T_{i}\right|_{\mathcal{M}_{*}}\right) X_{*}=X_{*} T_{i}, \quad i=1, \ldots, n .
$$

Indeed, since $\mathcal{M}_{*}$ is invariant under each $T_{i}^{*}, i=1, \ldots, n$, we have $P_{\mathcal{M}_{*}} T_{i} P_{\mathcal{M}_{*}}=$ $P_{\mathcal{M}_{*}} T_{i}, i=1, \ldots, n$. Using this relation together with (4.1) and (4.4), we infer that, for any $i=1, \ldots, n$,

$$
\begin{aligned}
X_{*} T_{i} & =P_{\mathcal{M}_{*}} Z^{-1} X Z T_{i}=P_{\mathcal{M}_{*}} Z^{-1} X T_{i} Z \\
& =P_{\mathcal{M}_{*}} Z^{-1}\left(\left.P_{\mathcal{M}} T_{i}\right|_{\mathcal{M}}\right) X Z=P_{\mathcal{M}_{*}} Z^{-1} T_{i} X Z \\
& =P_{\mathcal{M}_{*}} T_{i} Z^{-1} X Z=P_{\mathcal{M}_{*}} T_{i} P_{\mathcal{M}_{*}} Z^{-1} X Z \\
& =P_{\mathcal{M}_{*}} T_{i} X_{*} .
\end{aligned}
$$

According to (4.6), the noncommutative commutant lifting theorem, and relation (4.5), we find $Y_{*} \in \operatorname{Dil}_{\mathcal{M}_{*}}\left(X_{*}\right)$ satisfying

$$
\left\|Y_{*}\right\|=\left\|X_{*}\right\| \leq \rho_{\mathcal{M},\left\{T_{1}, \ldots, T_{n}\right\}^{\prime}}(X)+\epsilon .
$$

Set $Y:=W Y_{*} W^{-1}$ and let us show that $Y \in \operatorname{Dil}_{\mathcal{M}}(X)$. Notice that

$$
X=P_{\mathcal{M}} Z X_{*} Z^{-1} \text {. }
$$

Indeed, using (4.4), we have

$$
\begin{aligned}
P_{\mathcal{M}} Z X_{*} Z^{-1} & =P_{\mathcal{M}} Z\left(P_{\mathcal{M}_{*}} Z^{-1} X Z\right) Z^{-1}=P_{\mathcal{M}} Z P_{\mathcal{M}_{*}} Z^{-1} X \\
& =P_{\mathcal{M}} Z Z^{-1} X=P_{\mathcal{M}} X=X .
\end{aligned}
$$

Since $P_{\mathcal{M}_{*}} Y_{*}=X_{*} P_{\mathcal{H}}, Z^{-1}=\left.P_{\mathcal{H}} W^{-1}\right|_{\mathcal{H}}$, and $Y(\mathcal{K} \ominus \mathcal{H}) \subseteq \mathcal{K} \ominus \mathcal{H}$, we can use relation (4.8) to obtain

$$
\begin{aligned}
X P_{\mathcal{H}} & =P_{\mathcal{M}} Z X_{*} Z^{-1} P_{\mathcal{H}}=P_{\mathcal{M}} Z P_{\mathcal{M}_{*}} Y_{*} Z^{-1} P_{\mathcal{H}} \\
& =P_{\mathcal{M}} Z P_{\mathcal{H}} Y_{*} Z^{-1} P_{\mathcal{H}}=P_{\mathcal{M}}\left(\left.P_{\mathcal{H}} Z\right|_{\mathcal{H}}\right)\left(\left.P_{\mathcal{H}} Y_{*}\right|_{\mathcal{H}}\right)\left(\left.P_{\mathcal{H}} W^{-1}\right|_{\mathcal{H}}\right) P_{\mathcal{H}} \\
& =P_{\mathcal{M}}\left(\left.P_{\mathcal{H}} W Y_{*} W^{-1}\right|_{\mathcal{H}}\right) P_{\mathcal{H}}=P_{\mathcal{M}} Y P_{\mathcal{H}}=P_{\mathcal{M}} Y .
\end{aligned}
$$

According to (4.7), we have $\left\|W^{-1} Y W\right\|=\left\|Y_{*}\right\| \leq \rho_{\mathcal{M},\left\{T_{1}, \ldots, T_{n}\right\}^{\prime}}+\epsilon$. Hence $\rho_{\left\{V_{1}, \ldots, V_{n}\right\}^{\prime}}(Y) \leq \rho_{\mathcal{M},\left\{T_{1}, \ldots, T_{n}\right\}^{\prime}}(X)+\epsilon$ and $t \leq \rho_{\mathcal{M},\left\{T_{1}, \ldots, T_{n}\right\}^{\prime}}(X)+\epsilon$. This completes the proof.

The following result is a spectral version of the tangential Nevanlinna-Pick interpolation problem for $F_{n}^{\infty}$ (see [Po8]).

Theorem 4.2. Let $\lambda_{j}, j=1, \ldots, k$, be distinct elements in $\mathbb{B}_{n}$ and let $\mathcal{K}$ be a finite dimensional Hilbert space. If $u_{1}, \ldots, u_{k}, v_{1}, \ldots, v_{k} \in \mathcal{K}$ with $u_{i} \neq 0, j=1, \ldots, k$, and $\delta>0$, then there exists $\Phi \in F_{n}^{\infty} \bar{\otimes} B(\mathcal{K})$ such that

$$
\Phi\left(\lambda_{j}\right)^{*} u_{j}=v_{j}, j=1, \ldots, k, \quad \text { and } \quad \rho_{F_{n}^{\infty} \bar{\otimes} B(\mathcal{K})}(\Phi)<\delta
$$


if and only if there exist invertible operators $Z_{j} \in B(\mathcal{K}), j=1, \ldots, k$, such that

$$
\left[\frac{\left\langle\delta Z_{j} u_{j}, \delta Z_{i} u_{i}\right\rangle-\left\langle Z_{j} v_{j}, Z_{i} v_{i}\right\rangle}{1-\left\langle\lambda_{j}, \lambda_{i}\right\rangle}\right]_{1 \leq i, j \leq k}>0 .
$$

Proof. Let $\mathcal{N}:=\operatorname{span}\left\{z_{\lambda_{j}}: j=1, \ldots, k\right\}$ and $\mathcal{M}:=\mathbb{C} z_{\lambda_{1}} \otimes u_{1}+\cdots+\mathbb{C} z_{\lambda_{k}} \otimes u_{k}$ be a subspace of $\mathcal{N} \otimes \mathcal{K}$. Define $X\left(\left\{\lambda_{j}\right\},\left\{u_{j}\right\},\left\{v_{j}\right\}\right) \in B(\mathcal{N} \otimes \mathcal{K}, \mathcal{M})$ by setting $X\left(\left\{\lambda_{j}\right\},\left\{u_{j}\right\},\left\{v_{j}\right\}\right)^{*}\left(z_{\lambda_{j}} \otimes u_{j}\right):=z_{\lambda_{j}} \otimes v_{j}, j=1, \ldots, k$. For each $i=$ $1, \ldots, n$, define $T_{i}:=\left.P_{\mathcal{N}} S_{i}\right|_{\mathcal{N}} \otimes I_{\mathcal{K}}$ and notice that $T_{i}^{*} X^{*}=\left.X^{*} T_{i}^{*}\right|_{\mathcal{M}}$, where $X:=X\left(\left\{\lambda_{j}\right\},\left\{u_{j}\right\},\left\{v_{j}\right\}\right)$. Hence, $X T_{i}=P_{\mathcal{M}} T_{i} X$ for any $i=1, \ldots, n$.

As in the proof of Theorem 3.3, the minimal isometric dilation of the sequence $\left[T_{1}, \ldots, T_{n}\right]$ is $\left[S_{1} \otimes I_{\mathcal{K}}, \ldots, S_{n} \otimes I_{\mathcal{K}}\right]$ and $\left[F^{2}\left(H_{n}\right) \otimes \mathcal{K}\right] \ominus[\mathcal{N} \otimes \mathcal{K}]$ is hyperinvariant for $\left\{S_{1} \otimes I_{\mathcal{K}}, \ldots, S_{n} \otimes I_{\mathcal{K}}\right\}$. Since $\mathcal{M} \subseteq \mathcal{N} \otimes \mathcal{K}$ is invariant under each $T_{i}^{*}, i=1, \ldots, n$, we can apply Theorem 4.1 and infer that

$$
\rho_{\mathcal{M},\left\{T_{1}, \ldots, T_{n}\right\}^{\prime}}(X)=\inf \left\{\rho_{\left\{S_{1} \otimes I_{\mathcal{K}}, \ldots, S_{n} \otimes I_{\mathcal{K}}\right\}^{\prime}}(Y): Y \in \operatorname{Dil}_{\mathcal{M}}(X)\right\} .
$$

Since $\left\{S_{1} \otimes I_{\mathcal{K}}, \ldots, S_{n} \otimes I_{\mathcal{K}}\right\}^{\prime}=U^{*} F_{n}^{\infty} U \bar{\otimes} B(\mathcal{K})$, we can see that

$$
\rho_{\mathcal{M},\left\{T_{1}, \ldots, T_{n}\right\}^{\prime}}(X)<\delta
$$

if and only if there exists $\Phi \in F_{n}^{\infty} \bar{\otimes} B(\mathcal{K})$ such that $\rho_{F_{n}^{\infty} \bar{\otimes} B(\mathcal{K})}(\Phi)<\delta$ and

$$
P_{\mathcal{M}}\left(U^{*} \otimes I\right) \Phi(U \otimes I)=X P_{\mathcal{N} \otimes \mathcal{K}}
$$

Notice that

$$
\begin{aligned}
\left.\left\langle P_{\mathcal{M}}\left(U^{*} \otimes I\right) \Phi(U \otimes I)\left(z_{\lambda_{i}} \otimes k\right), z_{\lambda_{j}} \otimes u_{j}\right)\right\rangle & \left.=\left\langle\Phi\left(z_{\lambda_{i}} \otimes k\right), z_{\lambda_{j}} \otimes u_{j}\right)\right\rangle \\
& =\left\langle z_{\lambda_{i}}, z_{\lambda_{j}}\right\rangle\left\langle\Phi\left(\lambda_{j}\right) k, u_{j}\right\rangle \\
& =\left\langle z_{\lambda_{i}}, z_{\lambda_{j}}\right\rangle\left\langle k, \Phi\left(\lambda_{j}\right)^{*} u_{j}\right\rangle
\end{aligned}
$$

and $\left\langle X\left(z_{\lambda_{i}} \otimes k\right), z_{\lambda_{j}} \otimes u_{j}\right\rangle=\left\langle z_{\lambda_{i}}, z_{\lambda_{j}}\right\rangle\left\langle k, v_{j}\right\rangle$ for any $k \in \mathcal{K}$ and $i, j=1, \ldots, k$. Therefore, the relation (4.11) holds if and only if $\Phi\left(\lambda_{j}\right)^{*} u_{j}=v_{j}, j=1, \ldots, k$. On the other hand, if $Z \in\left\{T_{1}, \ldots, T_{n}\right\}^{\prime}$ then

$$
Z^{*}\left(z_{\lambda_{j}} \otimes k\right)=z_{\lambda_{j}} \otimes Z_{j} k, \quad k \in \mathcal{K},
$$

for some $Z_{j} \in B(\mathcal{K}), j=1, \ldots, k$. Notice that $Z$ is invertible if and only if $Z_{j}$ is invertible for any $j=1, \ldots, k$. Moreover, using the definition of $X=$ $X\left(\left\{\lambda_{j}\right\},\left\{u_{j}\right\},\left\{v_{j}\right\}\right)$ and (4.12), we have

$$
\left.Z^{*} X^{*}\left(\left\{\lambda_{j}\right\},\left\{u_{j}\right\},\left\{v_{j}\right\}\right) Z^{*-1}\right|_{Z^{*} \mathcal{M}}=X^{*}\left(\left\{\lambda_{j}\right\},\left\{Z_{j} u_{j}\right\},\left\{Z_{j} v_{j}\right\}\right) .
$$

Therefore,

$$
\rho_{\mathcal{M},\left\{T_{1}, \ldots, T_{n}\right\}^{\prime}}(X)=\inf \left\{\left\|X\left(\left\{\lambda_{j}\right\},\left\{Z_{j} u_{j}\right\},\left\{Z_{j} v_{j}\right\}\right)\right\|: Z_{j} \in B(\mathcal{K}) \text { are invertible }\right\}
$$

and relation (4.10) holds if and only if there exist invertible operators $Z_{j} \in B(\mathcal{K})$ such that $\left\|X\left(\left\{\lambda_{j}\right\},\left\{Z_{j} u_{j}\right\},\left\{Z_{j} v_{j}\right\}\right)\right\|<\delta$. This inequality is equivalent to

$$
\delta^{2} I-X\left(\left\{\lambda_{j}\right\},\left\{Z_{j} u_{j}\right\},\left\{Z_{j} v_{j}\right\}\right) X^{*}\left(\left\{\lambda_{j}\right\},\left\{Z_{j} u_{j}\right\},\left\{Z_{j} v_{j}\right\}\right)>0,
$$

which is equivalent to (4.9). This completes the proof.

We remark that (4.9) can be replaced by relation (4.10). As a consequence of Theorem 4.2, when the distinct elements in $\mathbb{B}_{n}$ are $\bar{\lambda}_{j}, j=1, \ldots, k$, we infer the following spectral tangential interpolation result for matrix-valued bounded analytic functions in the unit ball of $\mathbb{C}^{n}$. 
Corollary 4.3. Let $\lambda_{j}, j=1, \ldots, k$, be distinct elements in $\mathbb{B}_{n}$ and let $\mathcal{K}$ be a finite dimensional Hilbert space. If $u_{1}, \ldots, u_{k}, v_{1}, \ldots, v_{k} \in \mathcal{K}$ with $u_{i} \neq 0, j=1, \ldots, k$, $\delta>0$, and there exist invertible operators $Z_{j} \in B(\mathcal{K}), j=1, \ldots, k$, such that

$$
\left[\frac{\left\langle\delta Z_{j} u_{j}, \delta Z_{i} u_{i}\right\rangle-\left\langle Z_{j} v_{j}, Z_{i} v_{i}\right\rangle}{1-\left\langle\lambda_{i}, \lambda_{j}\right\rangle}\right]_{1 \leq i, j \leq k}>0
$$

then there exists $F \in H^{\infty}\left(\mathbb{B}_{n}\right) \bar{\otimes} B(\mathcal{K})$ such that

$$
\sup _{\lambda \in \mathbb{B}_{n}}\|F(\lambda)\|_{s p}<\delta \quad \text { and } \quad F\left(\lambda_{j}\right) u_{j}=v_{j}, j=1, \ldots, k .
$$

Let us make some remarks on the dependence of $\rho_{\mathcal{M},\left\{T_{1}, \ldots, T_{n}\right\}^{\prime}}(X)$ on the given interpolation data. For each $m=1, \ldots, k$, we define

$$
\rho_{m}:=\inf \left\{\left\|X\left(\left\{\lambda_{j}\right\}_{j=1}^{m},\left\{Z_{j} u_{j}\right\}_{j=1}^{m},\left\{Z_{j} v_{j}\right\}_{j=1}^{m}\right)\right\|: Z_{j} \in B(\mathcal{K}) \text { are invertible }\right\} .
$$

A multivariable analogue of [BF, Proposition 4] holds. More precisely, one can prove that if $u_{k}$ and $v_{k}$ are linearly independent, then $\rho_{k-1}=\rho_{k}$. Indeed, suppose that $\rho_{k-1}<\rho_{k}$. Using Theorem 4.2, we find $\Phi \in F_{n}^{\infty} \bar{\otimes} B(\mathcal{K})$ such that $\rho_{F_{n}^{\infty} \bar{\otimes} B(\mathcal{K})}(\Phi)<$ $\rho_{k}$ and $\Phi\left(\lambda_{j}\right)^{*} u_{j}=v_{j}, j=1, \ldots, k-1$. We may suppose that $\Phi\left(\lambda_{k}\right)^{*} \notin \mathbb{C} I_{\mathcal{K}}$ because, otherwise, we can replace $\Phi$ by $\Phi+\Psi$ for some $\Psi \in F_{n}^{\infty} \bar{\otimes} B(\mathcal{K})$ satisfying $\Phi\left(\lambda_{j}\right)=0, j=1, \ldots, k-1$, and $\Psi\left(\lambda_{k}\right) \notin \mathbb{C} I_{\mathcal{K}}$. Since we can choose $\Psi$ with very small norm we have $\rho_{F_{n}^{\infty} \bar{\otimes} B(\mathcal{K})}(\Phi+\Psi)<\rho_{k}$.

Therefore, since $\Phi\left(\lambda_{k}\right)^{*} \notin \mathbb{C} I_{\mathcal{K}}$, there exist linearly independent vectors $u$ and $v$ such that $\Phi\left(\lambda_{k}\right)^{*} u=v$. Since $u_{k}, v_{k}$ are linearly independent, we can find $Z_{k} \in B(\mathcal{K})$ invertible with $Z_{k} u_{k}=u$ and $Z_{k} v_{k}=v$. Hence, we infer that $\rho_{k} \leq \rho_{F_{n}^{\infty} \bar{\otimes} B(\mathcal{K})}(\Phi)<$ $\rho_{k}$, which is a contradiction. Since $\rho_{k-1} \leq \rho_{k}$, we must have $\rho_{k-1}=\rho_{k}$. This shows that in Theorem 4.2 we can assume, without loss of generality, that $v_{j}=\mu_{j} u_{j}$, for some $\mu_{j} \in \mathbb{C}, \mu_{j} \neq 0, j=1, \ldots, k$. Similarly to [BF, Proposition 5], one can show that if $k \leq \operatorname{dim} \mathcal{K}$, then

$$
\rho_{k}=\max \left\{\left|\mu_{1}\right|, \ldots,\left|\mu_{k}\right|\right\} .
$$

The case when the number of dependent vector pairs $\left(u_{j}, v_{j}\right)$ exceeds the dimension of $\mathcal{K}$, and the problem of optimal solutions will be considered in a future paper.

\section{REFERENCES}

[AMc] J. Agler and J.E. Mc Carthy, Nevanlinna-Pick kernels and localization, preprint (1997). CMP 2000:17

[ArPo1] A. Arias and G. Popescu, Factorization and reflexivity on Fock spaces, Integr. Equat. Oper.Th. 23 (1995), 268-286.

[ArPo2] A. Arias and G. Popescu, Noncommutative interpolation and Poisson transforms, Israel J. Math. 115 (2000), 205-234.

[Arv] W.B. Arveson, Subalgebras of $C^{*}$-algebras III: Multivariable operator theory, Acta Math. 181 (1998), 159-228. MR 2000e:47013

[BTV] J.A. Ball, T.T.Trent, and V. Vinnikov, Interpolation and commutant lifting for multipliers on reproducing kernel Hilbert spaces, preprint (1999).

[BV] J.A. Ball and V. Vinnikov, Multivariable linear systems, scattering, unitary dilations and operator theory for row contractions, preprint (1999).

[B] J.A. Ball, Linear systems, operator model theory and scattering: multivariable generalizations, preprint (1999).

[BFT] H. Bercovici, C. Foias, and A. Tannenbaum, A spectral commutant lifting theorem, Trans. Amer. Math. Soc. 325 (1991), 741-763. MR 91j:47006

[BF] H. Bercovici and C. Foias, On spectral tangential Nevanlinna-Pick interpolation, J.Math. Anal.Appl. 155 (1991), 156-176. MR 92d:47020 
[DP1] K.R. Davidson and D. Pitts, Invariant subspaces and hyper-reflexivity for free semigroup algebras, Proc. London Math.Soc., 78 (1999), 401-430.

[DP2] K.R. Davidson and D. Pitts, The algebraic structure of non-commutative analytic Toeplitz algebras, Math. Ann. 311 (1998), 275-303. MR 20001e:47082

[DP3] K.R. Davidson and D. Pitts, Nevanlinna-Pick interpolation for noncommutative analytic Toeplitz algebras, Integr. Equat. Oper.Th. 31 (1998), 321-337. MR 2000g:47016

[DMP] R.G. Douglas, P.S. Muhly and C. Pearcy, Lifting commuting operators, Michigan Math.J. 15 (1968), 385-395. MR 38:5046]

[F] I.P. Fedcina, A description of of the solutions of the Nevanlinna-Pick tangent problem, Akad. Nauk. Armjan. SSR Dokl. 60 (1975), 37-42. MR 52:5974

[N] R. Nevanlinna, Über beschränkte Functionen, die in gegebenen Punkten vorgeschribene Werte annehmen, Ann. Acad. Sci. Fenn. Ser A 13 (1919), 7-23.

[P] G. Pick, Über die Beschränkungen analytischer Functionen, welche durch vorgegebene Functionswerte bewirkt werden, Math. Ann. 77 (1916), 7-23.

[Po1] G. Popescu, Isometric dilations for infinite sequences of noncommuting operators, Trans. Amer.Math.Soc. 316 (1989), 523-536. MR 90c:47006

[Po2] G. Popescu, Characteristic functions for infinite sequences of noncommuting operators, J.Operator Theory 22 (1989), 51-71. MR 91m:47012

[Po3] G. Popescu, Von Neumann inequality for $\left(B(H)^{n}\right)_{1}$, Math.Scand. 68 (1991), 292-304. MR 92k:47073

[Po4] G. Popescu, On intertwining dilations for sequences of noncommuting operators, J.Math. Anal.Appl. 167 (1992), 382-402. MR 93e:47012

[Po5] G. Popescu, Functional calculus for noncommuting operators, Michigan Math. J. 42 (1995), 345-356. MR 96k:47025

[Po6] G. Popescu, Multi-analytic operators on Fock spaces, Math. Ann. 303 (1995), 31-46. MR 96k:47049

[Po7] G. Popescu, Noncommutative disc algebras and their representations, Proc. Amer. Math. Soc. 124 (1996), 2137-2148. MR 96k:47077

[Po8] G. Popescu, Interpolation problems in several variables, J.Math Anal.Appl. 227 (1998), 227-250. MR 99i:47028

[Po9] G. Popescu, Poisson transforms on some $C^{*}$-algebras generated by isometries, J. Funct. Anal. 161 (1999), 27-61. MR 2000m:46117

[R] G.C. Rota, On models for linear operators, Comm.Pure Appl.Math. 13 (1960), 469-472. MR 22:2898

[S] D. Sarason, Generalized interpolation in $H^{\infty}$, Trans. AMS 127 (1967), 179-203. MR 34:8193

[SzF1] B.Sz.-Nagy, C. Foiaş, Dilation des commutants d'operateurs, C.R.Acad.Sci. Paris, Serie A 266 (1968), 493-495. MR 38:5049

[SzF2] B.Sz.-Nagy, C. Foiaş, Harmonic analysis on operators on Hilbert space, North-Holland, Amsterdam (1970). MR 43:947

Division of Mathematics and Statistics, The University of Texas at San Antonio, San Antonio, Texas 78249

E-mail address: gpopescu@math.utsa.edu 